\title{
¿Por qué nõt? \\ Negación como incompatibilidad y matrices no-deterministas
}

\author{
(1) Eliana Franceschini \\ Universidad de Buenos Aires, Argentina
}

Recibido el 20/1/2018; aceptado el 5/3/2018.

\begin{abstract}
Resumen
El objetivo de este trabajo es reflexionar acerca del sentido de la negación, y defender que la mejor manera de caracterizar esta noción es a partir de la idea de incompatibilidad, que nos conduce a considerar la negación como un dispositivo de expresión de alguna forma de exclusión. Mostraré también que puede concebirse de esta manera a la negación sin necesidad de, como hacen Berto y Restall (2019), tener que aceptar que se trate de un operador modal. Para esto presentaré las matrices algebraicas no deterministas y explicaré cómo puede modelarse una negación que exprese incompatibilidades en contextos multivaluados sin dejar de ser una conectiva extensional.
\end{abstract}

\section{Why nõt? Negation as incompatibility and non-deterministic matrices}

\begin{abstract}
Abastract
The aim is to present a number of thoughts about the meaning of negation. I argue that the best characterization of this concept is grounded in the notion of incompatibility, which compels us to considerate negation as an exclusion-expressing device. I show that is possible to conceive negation in this way without having to accept, as Berto $\mathcal{E}$ Restall do, that it is a modal operator. For this I introduce algebraic non-deterministic matrices and explain how them allow us to model an exclusion-expressing device in the context of many-valued semantics, without having a negation that is not any more an extensional operator.
\end{abstract}

\section{Introducción}

La negación es una de las nociones lógicas centrales. Fue estudiada desde la antigüedad, siendo objeto de investigaciones exhaustivas en el desarrollo de disciplinas como la lógica filosófica, la lingüística, la inteligencia artificial y la lógica de la programación.

\section{Palabras clave}

$\overline{\text { Incompatibilidad }}$ lógica multivaluada negación semántica no-determinista

Keywords

Incompatibility multivalued logic negation non-deterministic semantics 
Las propiedades de la negación -en combinación con algunas de las otras operaciones lógicas y las características estructurales de la relación de consecuencia lógica- funcionan como portal entre diferentes sistemas lógicos. Es por esto que desempeña un rol muy importante en la selección de sistemas para diferentes aplicaciones particulares.

La negación es, en primer lugar, un fenómeno de oposición semántica. Como tal, relaciona una expresión A con otra expresión cuyo significado es de alguna manera opuesto al significado de A. Esta relación puede ser articulada de diversas maneras, tanto sintácticas como semánticas, en particular porque existen diferentes clases de oposición semántica.

La aparente simplicidad sintáctica de la negación disimula un tanto la profunda complejidad de su comportamiento, dadas las múltiples opciones lingüísticas que pueden utilizarse para expresar esta noción en el lenguaje natural: adverbios, verbos, cópulas, cuantificadores, afijos, etc. Los modos en los que diferentes lógicas seleccionan ciertos aspectos, a los fines de construir una conectiva que opere estructuralmente de manera similar (en algún sentido de similar) a la negación del lenguaje natural no son siempre sencillos y dan lugar a innumerables objeciones, evaluaciones y análisis críticos.

En primer lugar, bien puede peguntarse si es correcto hablar de "negaciones", en plural, o si existe una sola. No cabe duda de que distintos sistemas lógicos tienen, en muchos casos, negaciones diferentes. Pero, ¿es esto correcto?, ¿hay algo así como una negación ideal, que reúna un cúmulo de características que una negación debería tener para ser tal?, ¿qué tipo de operación es una negación?

Desde los escritos de Aristóteles sobre lógica (Aristóteles, Cat. y De Int.), hasta el siglo XXI los lógicos se plantean y tratan de responder cuestiones como las anteriores. Mientras para Aristóteles la negación es parte de un predicado (negativo), y por ende, opera en el interior de una proposición, la lógica de los estoicos o la de Frege (Frege, 1892) conciben a la negación como una conectiva unaria que se aplica a proposiciones formadas.

Usualmente se define como negación clásica aquella que valida las leyes de Explosión y de Tercero Excluído, y se considera paraconsistente una negación cuando no valida el primero de estos principios y paracompleta cuando hace fallar al segundo.

A los fines de responder interrogantes como los planteados anteriormente pueden utilizarse diversos enfoques modelo-teóricos. Puede considerarse, como en ocasiones F. Berto y G. Restall (2019), que la negación es una conectiva modal y que, por lo tanto, el mejor modo de expresar su significado es a través de una semántica kripkeana de mundos posibles. Si, como Frege, se la considera una conectiva unaria que opera alterando el valor de verdad de fórmulas de un lenguaje, entonces un modelo en términos de tablas de verdad resulta útil para su caracterización. $\mathrm{O}$, incluso, podríamos enfocarnos en los aspectos puramente sintácticos, como hace Avron (1999: 3-22) en algunas secciones de sus artículos y dar una descripción de la negación mediante una semántica de teoría de la prueba (proof-theoretic semantic). Por último, alguien como Priest (1987; 1999: 101-120), interesado en desarrollar técnicas para manejar información inconsistente estudiará alguna forma de operar con una negación que se adapte (o ayude a construir) algún contexto de paraconsistencia.

El objetivo del presente trabajo es analizar algunas consecuencias que se siguen, en contextos multivaluados, de aceptar que la mejor caracterización de la negación es aquella que la considera un dispositivo para expresar la exclusión. En lógica clásica los valores de verdad son sólo dos: lo verdadero y lo falso, y en virtud de esto, como bien señalan Szmuc y Omori (2017) sucede que muchas características de la negación permanecen 
ocultas. Cuando se incorpora, como sucede en sistemas paraconsistentes o paracompletos, más valores de verdad, comienza a ser necesaria una investigación más profunda de las propiedades tanto semánticas como sintácticas de la negación, así como también de cuáles son los efectos de priorizar ciertas características de la conectiva por sobre otras.

La tesis fundamental aquí será que, si se entiende la negación como un dispositivo de exclusión y se pretende incorporarla a un sistema con más de dos valores de verdad, la consecuencia es que el resultado de su empleo nos arroja un valor no determinista. Para introducir y explicar esta posición partiré, primero, de un recorrido general por aquel conjunto de características que usualmente se acuerda que una conectiva debe reunir para poder ser considerada una negación. Luego realizaré una breve exposición de los planteos de Berto y Restall (2019), dado que estos autores presentan una serie de argumentos para fundamentar la idea de que la mejor caracterización de la negación es aquella que la considera un dispositivo de exclusión. Asumiendo un punto de partida coincidente con el de estos autores mostraré que si lo que se excluye al utilizar la negación son los valores de verdad de un sistema (entre sí, es decir si se excluyen recíprocamente) y el sistema no es bivaluado, entonces los outputs de la conectiva negación no son "simples". Y para poder modelar una negación que se comporte así recurriré a una presentación breve de las matrices no deterministas, que considero son una herramienta algebraica útil para expresar la perspectiva propuesta.

Luego de una explicación, superficial, del funcionamiento de las n-matrices propondré tres opciones de negaciones no deterministas para contextos multivaluados. Finalmente realizaré una evaluación del recorrido del trabajo y señalaré la motivación para construir sistemas lógicos a partir de la incorporación de dichas negaciones a sistemas paraconsistentes $\mathrm{y} / \mathrm{o}$ paracompletos.

De los modos de presentación posibles de una lógica voy a elegir el enfoque modelo teórico, es decir, daré las cláusulas para las conectivas y definiré las distintas nociones de consecuencia lógica con las que opere en términos de valuaciones.

\section{Sistemas multivaluados}

Las reflexiones que propongo en torno a la conectiva negación se presentan dentro del marco de lo que se conoce como lógicas multivaluadas, esto es, sistemas que aceptan que sus fórmulas pueden tener más de dos valores de verdad. Estos sistemas fueron pensados y construidos con distintos fines y surgen de diversas motivaciones, que pueden ir desde un intento de evitar las paradojas semánticas que se producen al incorporar un predicado de verdad transparente a un sistema de lógica clásica, hasta la necesidad de operar formalmente con información inconsistente en grandes bases de datos.

Así, se abren diversos caminos posibles. Aquellos que abandonan la lógica clásica por la razón que fuere, pero acompañados de una desconfianza respecto del principio del Tercero Excluido (LEM: $\vDash \varphi \vee \sim \varphi$ ), suelen optar por sistemas, conocidos como paracompletos, que no validan esta ley en virtud de aceptar que algunas fórmulas no son ni verdaderas ni falsas, y para expresar esto se les adjudica el valor $\mathrm{n}$ (none). Un ejemplo de estos sistemas es el sistema lógico de Kripke (1975: 690-716), conocido como $\mathrm{K}_{3}(\kappa)$, que utiliza el esquema de valuación de Kleene Fuerte para definir sus conectivas, más una noción de consecuencia lógica basada en la transmisión de Valor Designado del sistema. Los valores del esquema son $\mathrm{K}=\{1,0, \mathrm{n}\}$, donde 1 es lo verdadero, o lo falso y n puede considerarse como lo "indeterminado", o aquello que no tiene valor de verdad, un gap. El único valor designado del sistema es 1, por lo que la validez en el sistema puede definirse de la siguiente manera: 
Una manera de entender a qué se considera paracompleto es decir que un sistema en el que existe al menos una valuación en la que LEM recibe un valor no designado es paracompleto. Es interesante notar que en $\mathrm{K}_{3}$ todas las fórmulas reciben al menos una posible valuación en $n$, con lo cual el no hay fórmulas universalmente válidas en el sistema. Las conectivas de $\mathrm{K}_{3}$ pueden caracterizarse mediante las siguientes tablas de verdad:

\begin{tabular}{|c|c|c|c|c|c|c|c|c|c|c|c|c|c|}
\hline$\wedge$ & 1 & $\mathrm{n}$ & 0 & V & 1 & $\mathrm{n}$ & o & כ & 1 & $\mathrm{n}$ & 0 & $A$ & $\neg A$ \\
\hline 1 & 1 & $\mathrm{n}$ & 0 & 1 & 1 & 1 & 1 & 1 & 1 & $\mathrm{n}$ & 0 & 1 & 0 \\
\hline $\mathrm{n}$ & $\mathrm{n}$ & $\mathrm{n}$ & 0 & $\mathrm{n}$ & 1 & $\mathrm{n}$ & $\mathrm{n}$ & $\mathrm{n}$ & 1 & $\mathrm{n}$ & $\mathrm{n}$ & $\mathrm{n}$ & $\mathrm{n}$ \\
\hline 0 & 0 & 0 & 0 & 0 & 1 & $\mathrm{n}$ & $\mathrm{o}$ & 0 & 1 & 1 & 1 & 0 & 1 \\
\hline
\end{tabular}

1. Se conoce con el nombre de dialeteismo aquella perspectiva filosófica que consiste en afirmar que ciertas contradicciones pueden ser verdaderas.
Por otro lado, los enfoques paraconsistentes surgen de la intención de operar con premisas inconsistentes sin dar como resultado algo trivial. Si la lógica valida el principio de Explosión () entonces ante la aparición de una contradicción, todo se sigue, podemos derivar cualquier cosa. Un sistema se considera paraconsistente cuando no valida Explosión (o no valida alguna de las formas, posiblemente en el metalenguaje también, de Explosión, como sostienen Barrio y Da Re (2018: 151-170)), y de esta forma es posible aceptar premisas contradictorias sin trivialidad. Ya sea porque se pretende trabajar con información inconsistente, o porque se favorece una interpretación filosófica dialeteista ${ }^{1}$ los sistemas paraconsistentes son una herramienta formal interesante en el marco de las lógicas multivaluadas. Un caso de una lógica como estas es Logic of Paradox, LP, defendida sobre todo por Priest (1987). La lógica LP $(\pi)$ utiliza también el esquema de valuación de Kleene Fuerte para definir sus conectivas, más una noción de consecuencia lógica basada en la transmisión de Valor Designado del sistema. Los valores del esquema son $K=\{1,0, b\}$, donde 1 es lo verdadero, o lo falso y b puede considerarse como lo "verdadero y falso", un glut. Los valores designados del sistema son $1 \mathrm{y} \mathrm{b}$, por lo que la validez en el sistema puede definirse de la siguiente manera:

DEFINICIÓN 2: (Válido en $L P$ ) : $\Gamma \vDash_{\pi}$ A sii en todo modelo $M_{\pi}$ en que $V_{\mathrm{m}_{\pi}}(\boldsymbol{\Gamma})=$ $1, \mathrm{~V}(\mathrm{~A})=1$ o bien $(\mathrm{A})=\mathrm{b}$

Las conectivas de LP se expresan así:

\begin{tabular}{|c|c|c|c|c|c|c|c|c|c|c|c|c|c|}
\hline$\wedge$ & 1 & b & 0 & $V$ & 1 & b & 0 & כ & 1 & $b$ & 0 & $A$ & $\neg A$ \\
\hline 1 & 1 & b & 0 & 1 & 1 & 1 & 1 & 1 & 1 & b & 0 & 1 & 0 \\
\hline b & $b$ & $b$ & 0 & $b$ & 1 & b & D & b & 1 & D & $b$ & $b$ & b \\
\hline 0 & 0 & 0 & 0 & 0 & 1 & b & 0 & 0 & 1 & 1 & 1 & 0 & \\
\hline
\end{tabular}

\section{Características generales de una negación}

Dejando de lado la necesidad de tomar posición en la disputa respecto de si puede o no hablarse de "negaciones" en plural, en lo que sigue se presentan una serie de propiedades esenciales que una gran parte de la literatura sobre el tema acuerda en que deben ser cumplidas por una conectiva para que sea una verdadera negación (Priest, Routley y Norman, 1989: 613.26, Berto, 2006: 241-263, Lenzen, 1996: 37-58, 
Humberstone, 2011: 1163-1284, Dunn, 1999: 23-51). Algunas de dichas propiedades están vinculadas a la relación entre el dispositivo lingüístico con el que negamos en el lenguaje ordinario y la constante que representa una acción similar en un sistema lógico y otras a cuestiones formales.

En líneas generales, y sin extendernos demasiado sobre un tópico complejo como este, una negación es una conectiva unaria, esto es, que se aplica directamente a fórmulas, y el resultado de aplicarla es, algunas veces, un cambio en su valor de verdad. A nivel formal es importante que sea una conectiva veritativo-funcional, creadora de contradicciones, ${ }^{2}$ que sea contrapositiva, ${ }^{3}$ que valide la Introducción de la Doble Negación, y que sea una conectiva extensional, es decir, que su significado pueda ser expresado mediante un algoritmo. Vinculado a la noción de consecuencia lógica propia de cada sistema, existen principios lógicos que usualmente se consideran característicos del significado de la negación, como el Principio de no Contradicción (LNC), Explosión o LEM. También se vincula al significado de la negación las llamadas Leyes de De Morgan para la conjunción y la disyunción4. Cuando se abandona la lógica clásica adoptando algún sistema que no valide alguno de estos principios la discusión respecto de lo propio de una negación debe basarse en consideraciones que no dependan de ellos. Distintos autores adoptan diversos enfoques: Humberstone o Priest, por ejemplo, priorizan el ser un operador formador de contradicciones, y Lenzen hace una distinción interesante entre propiedades inadmisibles, propiedades dispensables y propiedades indispensables. Las propiedades indispensables son aquellas que una lógica debe satisfacer si su operador monádico ha de contar como una genuina negación, y para este autor Contraposición es el más importante de todos.

\section{Negación como dispositivo de exclusión}

Voy ahora a explorar la perspectiva que hallo más interesante. Si tuviera que realizar una distinción como la propuesta por Lenzen, mi apuesta sería la de defender la importancia de la noción de incompatibilidad para una caracterización del significado de la negación, y por esto consideraría como propiedad indispensable la de ser un dispositivo que exprese alguna forma de exclusión. En este sentido encuentro que la propuesta de Berto y Restall (2019), BER, es en gran medida un buen punto de partida para el desarrollo de las tesis que defiendo. Allí estos autores distinguen entre los planes "Americano" y "Australiano" para la negación, y presentan dos ideas como las fundamentales del Plan Australiano -que es el que ellos defienden-. La Idea 1 es la siguiente: pronunciamos afirmaciones de la forma " $\neg \mathrm{A}$ " con el propósito de dejar algo de lado. Esto es: para expresar una cierta exclusión o incompatibilidad. Y la Idea 2 consiste en afirmar que una concepción de la negación como fundada en las nociones de incompatibilidad o exclusión debe considerar que se trata de un operador modal.

BER consideran que hay dos modos de comprender la incompatibilidad, que defienden por separado cada uno de los autores en otros textos: a) uno vinculado a los actos de habla de rechazo y aceptación (Restall, 2005: 189-205), que puede entenderse, de un modo normativamente pragmático, como señalando que una afirmación consiste en adoptar una cierta postura y comprometerse con ella -y por ende, excluir otras opciones-; y b) otro, más realista o "metafísico" (Berto, 2006: 241-263), como expresando que una determinada cosa es el caso -y por ende excluyendo otros posibles "estados del mundo". ${ }^{6}$ En cualquier caso, argumentan en favor de una fundación de la noción de negación como un dispositivo que exprese la incompatibilidad o la exclusión. Sostienen que la pregunta relevante no es qué negación es la correcta, sino por qué existen negaciones, para qué usamos el operador -ubicuo en los lenguajes

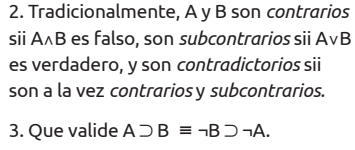

2. Tradicionalmente, $A$ y $B$ son contrarios sii $A \wedge B$ es falso, son subcontrarios sii $A v B$ es verdadero, y son contradictorios sii son a la vez contrariosy subcontrarios. 3. Que valide $A \supset B \equiv \neg B \supset \neg A$.

4. $\neg(A \vee B) \equiv(\neg A \wedge \neg B), y \neg(A \wedge B) \equiv(\neg A \vee \neg B)$.

5. Asumo que se refieren con el término "Americano" a "Norteamericano", en virtud de los autores que identifican con este plan.
6. Una discusión interesante respecto de este punto es la que desarrollan Rubin \& Roffé (2018: 189-202). Allí estos autores analizan los problemas de un enfoque metafísico de la noción de incompatibilidad. 
7. Consideran que algunas nociones, como identidad, referencia, realidad e incluso conjunto, son tan básicas para nuestra comprensión del lenguaje que todo intento de definición no puede sino ser de algún modo circular. Y esta circularidad no es para nada inaceptable, es una característica del modo en el que la mente humana comprende determinadas ideas, y es inevitable.
8. Para el lector interesado en profundizar este tema recomiendo De y Omori (2017). naturales- conocido como negación. Haciendo la salvedad de que "fundar" en este caso no va a poder ser equivalente a proporcionar una definición que reduzca de alguna manera el concepto a explicar a otras nociones que no contengan a su vez alguna forma del concepto explicado, 7 proceden a contestar la pregunta acerca de por qué existe algo así como la negación a partir de una reconstrucción del argumento de Price (1990: 221-238) que vincula fuertemente la idea de negación a la posibilidad de expresar incompatibilidades.

Price propone que nos imaginemos una comunidad, los Positivistas Ideológicos, en la que no existen negaciones y muestra de qué manera una comunidad de este tipo no podría expresar incompatibilidades. Y el sentido de lo incompatible proviene de nuestra experiencia del mundo en tanto agentes que enfrentan opciones: optar implica que existe una clase de lo posible que queda excluida. Necesitamos negaciones porque, incluso cuando pensamos, debemos poder expresar de alguna manera este fenómeno de lo incompatible. Y eso, tanto para Price, como para BER, es la característica más fundamental de la noción de negación. Ya sea en el enfoque a) o b), el modo en el que la incompatibilidad fundamenta el sentido de la negación es porque permite explicar la razón por la que tenemos algo así como el concepto de negación, permite contestar preguntas como: ¿por qué existen negaciones en todos los lenguajes naturales conocidos? Existe la negación porque la recíproca exclusión de cosas constituye una característica universal de la experiencia, y necesitamos un dispositivo lingüístico para expresar esa ubiquidad.

\section{"No es el caso" aplicado a valores de verdad}

Hasta aquí el desarrollo de la argumentación de BER resulta absolutamente acorde a las tesis que defiendo. Aceptando la Idea 1, voy mostrar que no necesariamente debemos optar por modelar una negación fundada en la noción de incompatibilidad utilizando semánticas kripkeanas de mundos posibles, como hacen estos autores. Parte de lo que motiva a estos autores a optar por considerar a la negación como un operador modal tiene que ver con la dificultad para articular una negación que funcione como un dispositivo de exclusión en el marco de lógicas distintas a la clásica, es decir, dar un enfoque tal que resulte lo suficientemente general para modelar la noción de negación propuesta sin necesidad de asumir un determinado framework. Con su semántica modal pueden caracterizar el comportamiento de la negación tanto en lógica clásica como en otros sistemas en los que los valores verdad y falsedad no sean mutuamente excluyentes o juntamente exhaustivos, pero esto al precio de que la negación ya no sea una conectiva veritativo funcional. Sin entrar en detalles acerca de los problemas de hacer de la negación un operador intensional, ${ }^{8}$ propongo una alternativa extensional con el mismo grado de generalidad y partiendo de la misma concepción de la negación como dispositivo de exclusión.

En lógica proposicional el significado de las proposiciones esta dado por su valor de verdad, es por esto que la semántica de un sistema se expresa a partir de la noción de valuación, que permite caracterizar el sentido de las constantes a partir de cláusulas sobre sus valuaciones. Por ejemplo, en lógica clásica las cláusulas que presentan el sentido de la negación son las siguientes: $\mathrm{v}(\neg \mathrm{A})=1$ sii $\mathrm{v}(\mathrm{A})=0, \mathrm{y} \mathrm{v}(\neg \mathrm{A})=0$ sii $\mathrm{v}(\mathrm{A})=$ 1. La negación clásica puede, como se ve, ser interpretada como aplicando la idea de "no es el caso" a los valores de verdad de las fórmulas sobre las que opera. ¿Qué ocurre si generalizamos esto para ser aplicado a contextos con más de dos valores de verdad? Si negar es, como sostengo más arriba, excluir algo, y si aquello que se decide excluir en particular son valores de verdad, ¿cómo funcionaría una negación que, al aplicarse a un determinado valor, excluyera los otros? 
En lógica clásica se solapan muchos atributos de la negación y se superponen interpretaciones posibles. La negación clásica nos da el complemento de lo negado, a la vez que puede interpretarse como un dispositivo de exclusión aplicado a valores de verdad. Si estas mismas características se insertan en sistemas como K3 o LP, lo que sucede es que, al multiplicarse los valores de verdad, se complejiza el output esperable de una negación como ésta. Como se observa en las tablas de verdad de los sistemas paraconsistentes o paracompletos presentados, sus negaciones son tales que nunca devuelven el complemento de lo negado, y por esto fallan como dispositivos de exclusión, si lo que se pretende es excluir recíprocamente valores de verdad. Y, dada la importancia, para la semántica de un sistema, de los valores de verdad, ¿no tendría sentido querer que la incompatibilidad que exprese la negación sea la de los valores de verdad entre sí? ¿qué otra cosa podría constituir un mejor candidato para expresar formalmente el sentido de la negación que su interacción con los sentidos -valores de verdad- de las fórmulas a las que se aplica?

\section{Matrices no deterministas}

Podría esperarse que no exista una manera veritativo funcional de expresar una noción de negación como esta en contextos multivaluados. Sin embargo, existe un tipo de matrices algebraicas provenientes de las ciencias de la computación que permiten construir semánticas capaces de modelar un dispositivo de exclusión de valores de verdad en espacios de valuación más complejos que la bivalencia clásica: las matrices no deterministas.

Pailos \& Rosenblatt (2015, 103: 579) realizan una presentación de estas matrices que puede resultar una buena introducción al tema, a ella recurro para mi descripción. Una semántica tiene una matrizno-determinista cuando está constituida por un triplo tal que $\mathrm{V}$ es un conjunto de valores de verdad, $\mathrm{D}$ es un subconjunto propio de $\mathrm{V}$ (los valores designados) y $\mathrm{O}$ es un conjunto de operaciones que representan el funcionamiento de las constantes del sistema. $Y$ debe existir al menos una constante $n$-aria que se defina mediante una función que toma como imagen un elemento que es un subconjunto de $\mathrm{V}$ y que no puede ser $\varnothing$.

DEFINICIÓN 3: (Matriz no determinista) Una matriz no determinista para un lenguaje $\mathcal{L}$ es un triplo $M=\langle V, D, O\rangle$, donde:

$V$ es un conjunto no vacío de valores de verdad,

$D$ es un subconjunto propio, no vacío, de $V, y$

$O$ es un conjunto de funciones tal que para cada conectiva n-aria $\otimes$ en $\mathcal{L}$, existe una correspondiente función $\otimes^{M}$ en tal que $\otimes^{M}: V^{n} \rightarrow P(V)-\varnothing^{9}$.

Esto significa que para que una semántica pueda considerarse no determinista debe existir al menos un operador cuyo resultado de aplicación sea más de un elemento del conjunto de valores de verdad. Esto no significa que el output sea complejo en un sentido "sincrónico", sino que la aplicación de la constante no determinista abre "caminos de valuaciones" que deben contemplarse todos como posibilidades, es decir, se amplía el conjunto de valuaciones relevantes. En una matriz determinista para cada conectiva n-aria $\otimes$ en $\mathcal{L}$, existe una función $\otimes^{M}$ tal que $\otimes^{M}: V^{n} \rightarrow V$. En el caso de las matrices no deterministas el codominio de la función correspondiente es el conjunto potencia del conjunto de valores $P(V)-\varnothing$, en lugar de ser el conjunto de valores. De esta forma se observa como las matrices deterministas son un caso de las no deterministas. Es decir, que para cada conectiva n-aria $\otimes$ en una matriz determinista $M$
9. La razón para excluir el conjunto vacío es que no está claro cómo computar el valor de fórmulas compuestas cuando se tiene como input el conjunto vacío. 
que sea interpretada como una función $\otimes^{M}: V^{n} \rightarrow V$, es posible construir una matriz no determinista $M$ ' donde esa conectiva sea considerada como una función que sólo devuelve un valor, esto es, $\otimes^{M^{\prime}}: V^{n^{\prime}} \rightarrow\{A \subseteq V:|A|=1\}$. De esta forma obtenemos una matriz no determinista con conectivas que copian el comportamiento de conectivas deterministas.

¿Qué cosas pueden ser modeladas con este tipo de matrices? Un ejemplo, que proporcionan Avron \&Zamansky (2008: 227-304), es el de la indeterminación lingüística de los sentidos de la disyunción en el lenguaje natural: cuando decimos "A o B" podemos querer decir: o bien que es una opción o la otra, pero no las dos (disyunción exclusiva), o bien que pueden también darse las dos opciones (inclusiva). Esta ambigüedad puede ignorarse, como sucede en la mayoría de los sistemas formales -donde se opta por una u otra alternativa- o modelarse mediante una tabla de verdad no determinista como la que sigue.

\begin{tabular}{ccc}
$V$ & 1 & 0 \\
\hline 1 & $\{1 ; 0\}$ & 1 \\
0 & 1 & 0
\end{tabular}

Finalmente, la noción de valuación puede caracterizarse de la siguiente manera para una semántica no determinista:

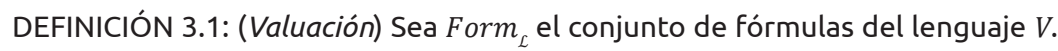
Una valuación en $M$ es una función $v:$ Form $_{\mathcal{L}} \rightarrow v$ tal que para cada conectiva n-aria $\otimes$ de $V$, lo siguiente se sostiene para toda $\varphi_{1}, \ldots \varphi_{n} \in$ Form $_{\mathcal{L}}: \mathcal{V}\left(\otimes\left(\varphi_{1}, \ldots\right.\right.$, $\left.\left.\varphi_{n}\right)\right) \in \otimes^{\mathfrak{M}}\left(\mathcal{V}\left(\varphi_{1}\right), \ldots, \mathcal{V}\left(\varphi_{n}\right)\right)$

Como $\otimes^{\mathcal{M}}\left(\mathcal{V}\left(\varphi_{1}\right), \ldots, \mathcal{V}\left(\varphi_{n}\right)\right.$ nos devuelve un conjunto de valores en lugar de un sólo valor, se usa en lugar de = en la definición. Los conceptos de satisfacción y validez pueden definirse del modo habitual.

Si lo que queremos es modelar una negación que pueda interpretarse como " no es el caso que x valor de verdad" en un sistema con más de dos valores de verdad, podemos utilizar una semántica no determinista. Y eso es lo que haré en la próxima sección de este trabajo.

\section{Ejemplos de lógicas multivaluadas + nõt}

En la parte 2 di tres ejemplos de lógicas multivaluadas: $\mathrm{K}_{3}$ y LP. Veamos ahora cómo podrían construirse opciones de negaciones no deterministas para estos sistemas. Llamaré nõt a la negación que propongo, y será una conectiva que opere en estos sistemas como dispositivo de exclusión. Lo que debería suceder es que en cada caso de aplicación de esta conectiva el resultado sea los valores de verdad del sistema menos el valor negado. Es decir, que si nos hallamos por ejemplo en un marco paraconsistente como LP, en el que los valores de verdad son 1 , o y b y queremos negar una fórmula cuyo valor es 1 , nuestra negación deberá poder expresar la idea de que existe una incompatibilidad entre el valor 1 y los otros dos valores del sistema. Dicho de otro modo, si a priori uno no sabe cuál es el valor de verdad de una fórmula que contiene una negación, pero sí sabe que la fórmula atómica, sin negación, tiene valor 1 entonces se deberá aceptar que la clase que queda excluida al negar es la que contiene a o y a b. La fórmula negada tendrá entonces cualquiera de esos dos valores, y cómo el resultado está indeterminado en el momento de 
aplicación de la conectiva -dadas las características del sistema, que presenta un espacio múltiple de valores de verdad- deberemos contemplar ambas opciones, esto es, agregar valuaciones posibles.

Mostremos entonces cómo serían las tablas de verdad de la negación para sistemas que tuvieran las mismas tablas de verdad para todas las conectivas que $\mathrm{K}_{3}$ y LP, pero reemplazando la negación de esas lógicas por nõt. En cada caso la noción de consecuencia lógica también se mantiene igual que en la lógica original.

EJEMPLO 1: (matriz no-determinista para un sistema como $\mathrm{K}_{3}+$ not -$\urcorner \mathrm{K}_{3}$ ) Sea $\mathcal{L}$ un lenguaje proposicional con una conectiva unaria nõt, y tres conectivas binarias $\Lambda, V$ y $\supset$. Sea $\mathcal{M}_{1}=\left\langle V_{1}, D_{1}, O_{1}\right\rangle$, donde:

$$
\begin{aligned}
& V_{1}=\{1 ; 0 ; n\}, \\
& D_{1}=\{1 ; n\}, y
\end{aligned}
$$

\begin{tabular}{|c|c|c|c|c|c|c|c|c|c|c|c|c|c|}
\hline$\Lambda^{\mathcal{M}_{1}}$ & 1 & $\mathrm{n}$ & 0 & $\mathrm{~V}^{\mathfrak{M}_{1}}$ & 1 & $\mathrm{n}$ & 0 & $\supset^{\mathcal{M}_{1}}$ & 1 & $\mathrm{n}$ & 0 & A & nõtA \\
\hline 1 & $\{1\}$ & $\{\mathrm{n}\}$ & $\{0\}$ & 1 & $\{1\}$ & $\{1\}$ & $\{1\}$ & 1 & $\{1\}$ & $\{n\}$ & $\{0\}$ & 1 & $\{0 ; n\}$ \\
\hline$n$ & $\{\mathrm{n}\}$ & $\{\mathrm{n}\}$ & $\{0\}$ & $\mathrm{n}$ & $\{1\}$ & $\{\mathrm{n}\}$ & $\{\mathrm{n}\}$ & $\mathrm{n}$ & $\{1\}$ & $\{n\}$ & $\{n\}$ & $n$ & $\{1 ; 0\}$ \\
\hline 0 & $\{0\}$ & $\{0\}$ & $\{0\}$ & 0 & $\{1\}$ & $\{\mathrm{n}\}$ & $\{0\}$ & 0 & $\{1\}$ & $\{1\}$ & $\{1\}$ & 0 & $\{1 ; n\}$ \\
\hline
\end{tabular}

$O_{1}$ se define de la siguiente manera:

El caso de LP es igual, pero el tercer valor es b en lugar de n y los valores designados del sistema son dos: $1 \mathrm{yb}$.

\section{Algunas propiedades de estos (proyectos de) sistemas}

Cada uno de los sistemas no deterministas resultantes tendrá diferentes características y validará ciertos principios y no otros. Para dar sólo algunos ejemplos, en LP + nõt el principio de Explosión (A nõt A B) no es universalmente válido porque existe al menos una valuación que da un valor designado a las premisas (b) y un valor no designado a la conclusión. Si tenemos A nõt A no nos es lícito inferir B dado que, por ejemplo, cuando A vale 1 tenemos que v(nõt A) puede ser b o o, dado que la tabla de verdad para nõt en este sistema es

$$
\begin{array}{cc}
\text { A } & \text { nõtA } \\
\hline 1 & \{0 ; b\} \\
\text { b } & \{1 ; 0\} \\
0 & \{1 ; b\}
\end{array}
$$

y con esto se da que

$$
\frac{\mathrm{A} \wedge}{1\{0 ; \mathrm{b}\}}\left\{\begin{array}{l}
\text { nõtA } \\
\{0 ; \mathrm{b}\}
\end{array}\right.
$$

y por lo tanto no se da que B se siga en todos los casos, porque existe al menos una valuación posible que le otorga un valor designado a las premisas. De este modo, la paraconsistencia del sistema original sería conservada en el nuevo sistema.

De nuevo en LP + nõt, el principio de Tercero Excluido resulta válido, dado que toda valuación de $\mathrm{A}$ le da un valor designado. Si v $(\mathrm{A})=1$ entonces el valor de nõt A será 
alguno de los elementos del conjunto $\{0 ; \mathrm{b}\}$ y con esto la valuación de $\mathrm{A} \vee \mathrm{n}$ nõt $\mathrm{A}$ será siempre 1, para cualquiera de los caminos que se abran cuando se opera con la conectiva no determinista del sistema, como puede observarse en la siguiente tabla:

$$
\begin{aligned}
& \text { A } \quad \text { V nõtA } \\
& 1\{1 ; 1\}\{0 ; b\}
\end{aligned}
$$

Si $\mathrm{v}(\mathrm{A})=0$ el valor de nõt $\mathrm{A}$ es o bien 1 o bien $\mathrm{b}$, con lo que $\mathrm{A} \vee$ nõt $\mathrm{A}$ recibe los siguientes valores:

$$
\begin{aligned}
& \text { A } V \text { nõtA } \\
& 0 \quad\{1 ; b\} \quad\{1 ; b\}
\end{aligned}
$$

Finalmente, si $\mathrm{v}(\mathrm{A})=\mathrm{b}$ el valor de nõt A es o bien 1 o bien $\mathrm{o}$, que son los otros dos valores disponibles en el sistema, y con esto resulta de nuevo que la disyunción $\mathrm{A}$ $V$ nõt A obtiene un valor designado para cada posible valuación en un modelo no determinista.

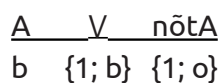

Otras características, en cambio, resultarán problemáticas en los nuevos sistemas: ninguna negación no determinista validará las leyes de De Morgan para una conjunción y una disyunción que interactúen con ella, y Doble Negación tampoco será una regla válida para una conectiva como nõt.

La doble negación falla porque al aplicar la negación cada vez se abren caminos no deterministas nuevos que nos devuelven más valuaciones posibles, con lo cual nunca ocurre que la valuación de A y de nõt nõt A sean iguales. Para ver en detalle lo que ocurre préstese atención a la siguiente tabla (que presenta los valores de la doble negación, con nõt en un sistema paracompleto):

$$
\begin{array}{ccc}
\text { A } & \text { nõtA } & \text { nõt nõtA } \\
\hline 1 & \{0 ; n\} & \{0 ; n ; 1 ; n\} \\
n & \{1 ; 0\} & \{0 ; n ; 1 ; n\} \\
0 & \{1 ; n\} & \{0 ; n ; 1 ; 0\}
\end{array}
$$

\section{Conclusión}

Se propuso, en el presente trabajo, una reflexión acerca del modo más adecuado de fundar el sentido de la negación. Defendí aquí, en sintonía con BER, que la noción de incompatibilidad es más importante para el concepto de negación que cualquier otra de las características defendidas por otros autores: la negación puede no ser cualquier cosa, pero no puede no funcionar como el dispositivo formal con el que se expresa alguna forma de exclusión. En el punto donde, de asumir esto, BER derivan la conclusión de que la mejor manera de modelar una negación así concebida es haciendo de ella un operador modal, me separo de ellos para proponer la utilidad de las matrices algebraicas no deterministas para construir semánticas que permitan expresar negaciones como dispositivos de exclusión al mismo tiempo que se conserva la extensionalidad de la conectiva.

Sería interesante emprender la construcción de algunos de los sistemas conjeturados a los fines de poder analizar en profundidad qué virtudes y defectos tienen marcos formales como esos, o cuáles podrían ser sus aplicaciones. Considero satisfactorio 
sin embargo el recorrido realizado, dado que fue posible cumplir con sus dos objetivos fundamentales: defender una cierta noción del significado de la negación y a la vez mostrarla no incompatible con su expresión como operador extensional. Por otra parte motivé la creación de, al menos, dos sistemas nuevos, con negaciones que expresan incompatibilidades de valores de verdad en contextos multivaluados. 


\section{Bibliografía}

» Aristóteles (1963). [Cat.] y [De Int.], Categories and De Interpretatione (ed. y trad. J. Ackrill). Oxford: Clarendon.

" Avron, A. (1999). Negation: Two Points of View. En Gabbay, D. \& Wansing H. (Eds). What is a Negation? (pp. 3-22). Applied Logic Series, 13. https://doi. org/10.1007/978-94-015-9309-0_1

»Avron, A. \& Zamansky, A. (2008). Non-deterministic semantics for logical systems. En Gabbay, D. and Guenthner, F. (Eds.). Handbook of Philosophical Logic, 16: 227304. Kluwer Academic Publisher. https://doi.org/10.1007/978-94-007-0479-4_4

" Berto, F. (2006). Characterizing negation to face dialetheism. En Logique \& Analyse, 49,(195), 241-263.

» Berto, F. \& Restall G. (2019). Negation on the Australian Plan. En Journal of Philosophical Logic. https://doi.org/10.1007/s10992-019-09510-2

» Barrio, E. \& Da Re, B. (2018). Paraconsistency and philosophical interpretations, Australasian Journal of Logic, 15 (2), 151-170.

»De, M. \& Omori H. (2017). Classical Negation and Expansions of Belnap-Dunn Logic. En Studia Logica, 103(4). http://dx.doi.org/10.1007/s11225-014-9595-7

" De, M \& Omori, H. (2017). There is More to Negation than Modality. En Journal of Philosophical Logic, 1-19. https://doi.org/10.1007/s10992-017-9427-0

" Dunn J. M. (1999). A Comparative Study of Various Model-theoretic Treatments of Negation: A History of Formal Negation. En Gabbay, D. \& Wansing H. (Eds). What is a Negation? (pp. 23-51). Applied Logic Series, 13. https://doi. org/10.1007/978-94-015-9309-0_2

»Frege, G. (1892). On Sinn and Bedeutung. En M. Beaney (Ed.). The Frege Reader, 151-71. Oxford: Blackwell.

» Humberstone, L. (2011). The connectives. The MIT Press, 1163-1284. https://doi.o $\mathrm{rg} / 10.1080 / 00048402.2013 .765901$

» Kripke, S. (1975). Outline of a theory of truth. Journal of Philosophy, 72(19): 690716.

"Lenzen, W. (1996). Necessary Conditions for Negation Operators. En Wansing, H. (Ed.). Negation. A Notion in Focus (37-58). Berlin: De Gruyter. https://doi. org/10.1007/978-94-017-1739-7_7

» Pailos F. \& Rosenblatt, L. (2015). Non-deterministic conditionals and transparent truth. Studia Logica, 103: 579. https://doi.org/10.1007/s11225-014-9580-1

» Price, H. (1990). Why 'not'?. Mind, 99: 221-238 https://doi.org/10.1093/mind/ XCIX.394.221

» Priest, G. (1987). In Contradiction: a Study of the Transconsistent. Dordrecht: Martinus Nijhoff.

» Priest, G. (1989). Reductio ad Absurdum et Modus Tollendo Ponens. En Priest, Routley \& Norman (Eds.). Paraconsistent Logic: Essays on the Inconsistent (pp. 613626). Munich: Philosophia Verlag.

" Priest, G (1999). What not? A Defence of Dialetheic Theory of Negation. En 
Gabbay, D. \& Wansing H. (Eds). What is a Negation? (pp 101-120). Applied Logic Series, 13. https://doi.org/10.1007/978-94-015-9309-0_6

»Priest, G. (2008). An Introduction to Non-Classical Logic. Nueva York: Cambridge University Press.

"Rofeé, A. y Da Re, B. (2016). Introducción a las lógicas no clásicas. Buenos Aires: OPFyL.

》 Restall, G. (2005). Multiple conclusions. En Hájek, P. Valdés-Villanueva, L \& Westerstahl, D. (Eds). Logic, Methodology and Philosophy of Science: Proceedings of the Twelfth International Congress, (p189-205). KCL Publications. http://consequently.org/writing/multipleconclusions.

" Rubin, M. \& Rofeé, A. (2018). Against a metaphysical understanding of rejection. Principia, 22 (1): 189-202. doi: 10.5007/1808-1711.2018v22n1p189

"Szmuc D, \& Omori H. (2017). Liberating classical negation from falsity conditions (Manuscrito inédito). 
